\title{
Installation of the IMPAC Technique in the Pelletron Laboratory
}

\author{
M.N. Rao, J.R.B. Oliveira, W.A. Seale, M.A. Rizzutto, R.V. Ribas, \\ J.A. Alcántara Núñez, D. Pereira, N. Added, E.W. Cybulska, N.H. Medina \\ Pelletron Laboratory, Dept. of Nuclear Physics, Institute of Physics, University of So Paulo, So Paulo, SP, Brazil
}

\author{
R.N. Saxena and A.W. Carbonari \\ Nuclear Physics and Chemistry Dept., IPEN-CNEN/SP
}

Received on 30 October, 2002

\begin{abstract}
A special target chamber for the direct production \& recoil implantation of radioactive probes for perturbed angular correlation has been constructed and tested. Feasibility studies were made by reproducing the known quadrupole frequencies at ${ }^{77} \mathrm{Br}$ and ${ }^{111} \mathrm{In}$ probes in metallic zinc. Preliminary results were obtained on the lattice site dependence of $\mathrm{Cd}$ hyperfine field in $\mathrm{Pd}_{2} \mathrm{MnSn}$ Heusler alloy with the PAC technique.
\end{abstract}

The Perturbed Angular Correlation (PAC) technique based on the hyperfine (HF) interactions of radioactive probe nuclei is a very useful tool in the study of magnetic hyperfine fields (MHF) and electric field gradients (EFG) at crystalline sites. In these experiments one observes the interaction of an internal magnetic field with the nuclear magnetic dipole moment or that of the electric field gradient with the nuclear quadrupole moment of an intermediate state of a gamma-gamma cascade in the probe nucleus. The interaction leads to a change in the gammagamma angular correlation between the two emitted gamma rays, which can be observed by recording time differential coincidence spectra $g g(t)$, with scintillation $(\mathrm{BaF} 2)$ detectors arranged in a plane around the sample. The coincidence spectra show the exponential decay of the intermediate state modulated by a perturbation function $G_{22}(t)$, which in the case of a magnetic interaction is given by $G_{22}(t)=$ $0.2\left[1+2 \cos \left(\omega_{L} t\right)+2 \cos \left(2 \omega_{L} t\right)\right]$, where $\omega_{L}=g \mu_{N} B_{h f} / \hbar$ is the Larmor frequency, and $g$ is the nuclear $g$ factor and $B_{h f}$ is the magnetic hyperfine field.

In the case of an electric quadrupole interaction, the corresponding perturbation factor is given by

$$
G_{22}(t)=S_{20}+\sum_{n=1}^{3} S_{2 n} \cos \left(\omega_{n} t\right) \exp \left(-\omega_{n}^{2} \tau_{R}^{2} / 2\right) \exp \left(-\omega_{n}^{2} \delta^{2} t^{2} / 2\right)
$$

where the primary frequencies $\omega_{n}$ and their amplitudes $S_{2 n}$ are related to the hyperfine splitting of the intermediate nuclear level and depend on the nuclear quadrupole frequency $\omega_{Q}=e Q V_{z z} / 4 I(2 I-1) \hbar$ and the asymmetry parameter $\eta=\left(V_{x x}-V_{y y}\right) / V_{z z}$ where $V_{x x}, V_{y y}$ and $V_{z z}$ are the elements of the EFG tensor in its principal axis system. As usual, $V_{z z}$ is the largest component of the EFG tensor and generally one uses the spin independent quadrupole frequency defined by $\nu_{Q}=e Q V_{z z} / h$, where $Q$ is the nuclear electric quadrupole moment of the intermediate level. The known quadrupole moment of $0.83 \mathrm{~b}$ for the $\mathrm{I}=5 / 2^{+}$intermediate level of ${ }^{111} \mathrm{Cd}$ has been used to determine $V_{z z}$. The effects of finite time resolution $\tau_{R}$ of detectors and the distribution of EFG with a width $\delta$ are properly taken into account in the above equantion.

From the perturbation pattern, the magnitude and the sign of the MHF, the magnitude, symmetry and the orientation of the EFG tensor or both may be determined. Very often, the hyperfine interactions corresponding to different crystalline sites are observed. In these cases, the perturbation factor $G_{22}(t)=\sum_{i} f_{i} G_{22}^{i}(t)$ takes into account the fractional site populations $f_{i}$ and $G_{22}^{i}(t)$ are the corresponding perturbation factors.

The introduction of the radioactive probes into the sample is usually done chemically, by diffusion or melting, or by ion implanter. In most cases, an annealing follows the chemical or melting process extending the sample preparation time, which makes the use of short half-life nuclei im- 
possible. Another technique used is to produce the probes directly in a nuclear reaction with an accelerated heavy-ion beam and implant them as they recoil out of the target, into the sample placed behind the target (Fig. 1).

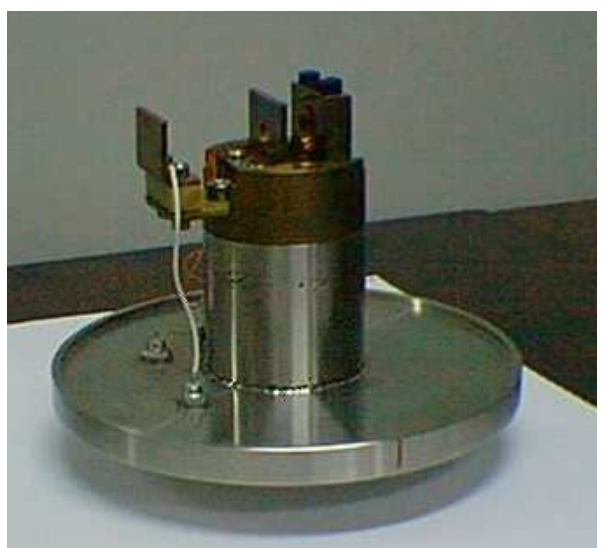

Figure 1. Experimental setup for production and recoil implantation of PAC probes.

This last method is expensive as one needs an accelerator, but highly efficient. In the case when the sample itself serves as the target the implantation process could be $100 \%$ efficient, i.e., all the probes produced could be implanted in the sample.

While the atomic binding energies in a crystal are of the order of $\mathrm{eV}$, the process of implantation involves $\mathrm{keV}$ to $\mathrm{MeV}$ energies and thus is accompanied by radiation damage. To eliminate or at least reduce this damage the samples have to be annealed after the implantation of the radioactive probes.

At the Pelletron laboratory, we have so far produced the radioactive ${ }^{77} \mathrm{Br}\left(\mathrm{T}_{1 / 2}=56 \mathrm{~h}\right)$ and ${ }^{111} \mathrm{In}\left(\mathrm{T}_{1 / 2}=2.83 \mathrm{~d}\right)$ PAC probes. The reactions 1 to produce these two probes were, respectively, ${ }^{68} \mathrm{Zn}\left({ }^{12} \mathrm{C}, 3 \mathrm{n}\right){ }^{77} \mathrm{Kr} \Rightarrow(1.2 \mathrm{~h}){ }^{77} \mathrm{Br}$ and ${ }^{68} \mathrm{Zn}$ $\left({ }^{12} \mathrm{C}, \mathrm{p} 2 \mathrm{n}\right){ }^{77} \mathrm{Br}$ at $\mathrm{E}_{\text {inc }}=54 \mathrm{MeV}$ and ${ }^{108} \mathrm{Pd}\left({ }^{7} \mathrm{Li}, 4 \mathrm{n}\right){ }^{111} \mathrm{In}$ $(2.83 \mathrm{~d})$, as well as ${ }^{108} \mathrm{Pd}\left({ }^{6} \mathrm{Li}, 3 \mathrm{n}\right){ }^{111} \mathrm{In}$ at $\mathrm{E}_{i n c}=32 \mathrm{MeV}$. The results obtained so far by way of feasibility studies are shown below. The quadrupole coupling constant obtained in metallic zinc at the site of the ${ }^{77} \mathrm{Br}$ probe was $\nu_{q}=92 \pm 5$ $\mathrm{MHz}$ with the assymetry parameter $\eta=0.2$ (Fig. 2).

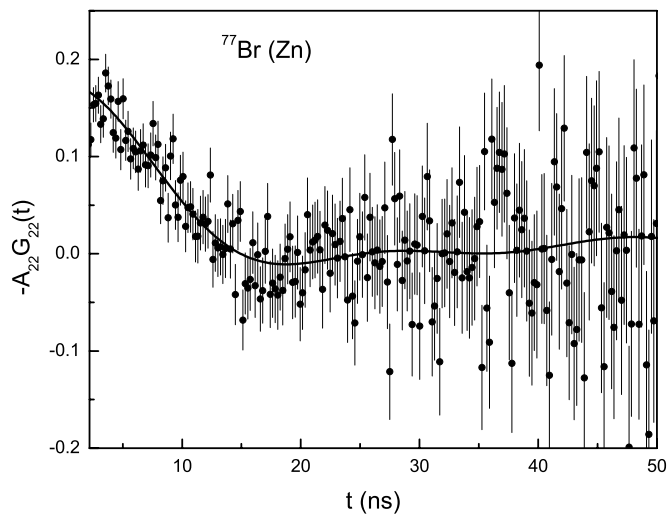

Figure 2. Measured ratio function following the implantation of metallic zinc samples with ${ }^{77} \mathrm{Br}$. and at the site of the ${ }^{111}$ In probe $\nu_{q}=133.0 \pm 1.5 \mathrm{MHz} ; \eta$ $=0$ (Fig. 3).

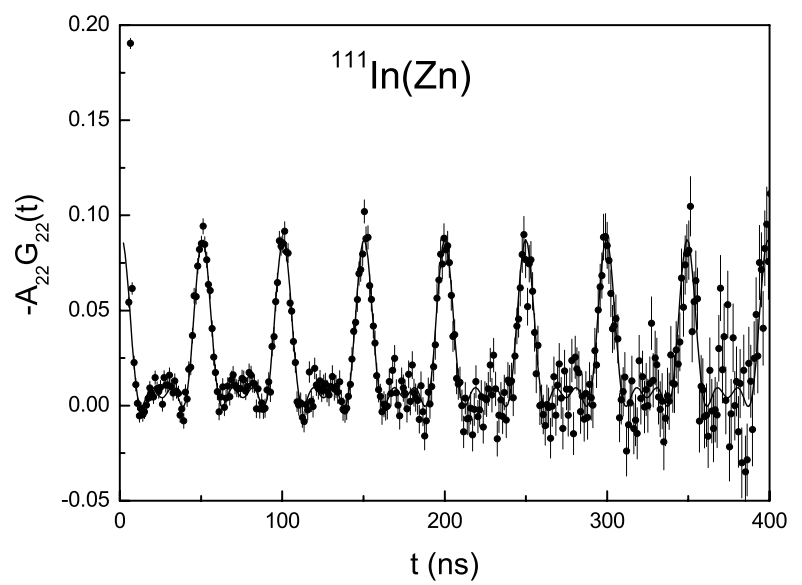

Figure 3. Measured ratio function following the implantation of metallic zinc samples with ${ }^{111}$ In.

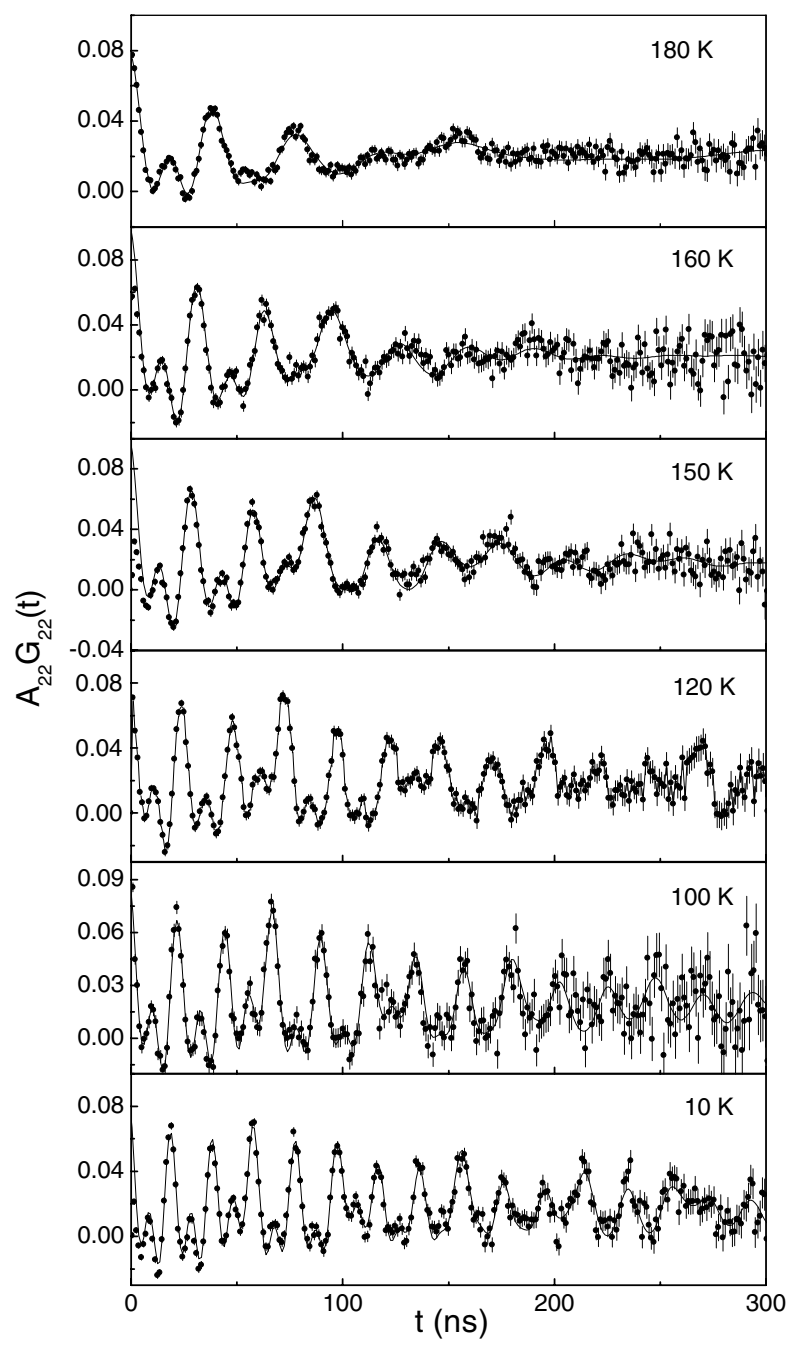

Figure 4. The pertubation functions for ${ }^{111}$ In probes in $\mathrm{Pd}_{2} \mathrm{MnSn}$ Heusler alloy at various temperatures. 
The above frequencies are in excellent agreement with known values from literature $[1,2,3,4]$ thus showing the feasibility of applying the IMPAC technique in the Pelletron laboratory.

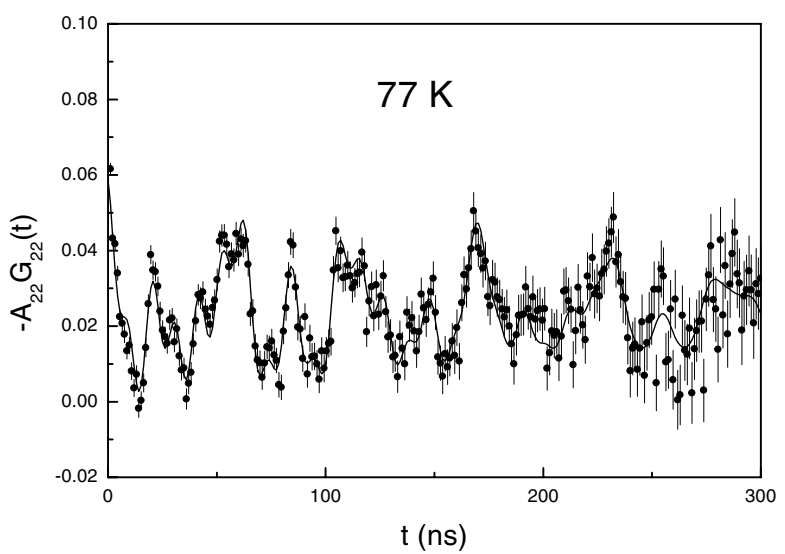

Figure 5. The perturbation functions for ${ }^{111}$ In probes in $\mathrm{Pd}_{2} \mathrm{MnSn}$ Heusler alloy at $77 \mathrm{~K}$ after additional annealing at $800{ }^{\circ} \mathrm{C}$.

We have now initiated a systematic study of the site dependence of the MHF in Heusler alloys with the IMPAC technique using the $4 \mathrm{BaF}_{2}$ PAC spectrometer of IPEN [5]. First measurements on the ternary alloy $\mathrm{Pd}_{2} \mathrm{MnSn}$ with ${ }^{111}$ In probes showed two Larmor frequencies corresponding to the $\mathrm{Mn}$ and $\mathrm{Sn}$ sites [6]. The temperature dependence of the perturbation function can be seen in Fig. 4, where the PAC spectra are characterized by sharp and well resolved frequencies. The samples were annealed at $400^{\circ} \mathrm{C}$ for about 4 hours after implantation before the PAC spectra were obtained. PAC spectrum at $77 \mathrm{~K}$ after an additional annealing of $800{ }^{\circ} \mathrm{C}$ for $8 \mathrm{~h}$ has shown an increase of the fraction corresponding to the Mn site (see Fig. 5). Further measurements to better understand the annealing temperature dependence of the ${ }^{111}$ In-site occupation as well as to obtain the MHF at the Pd site are planned.

\section{References}

[1] University of Bonn, Annual Report (1974).

[2] J. Physics F 4, L80 (1974).

[3] R.S. Raghavan, E.N. Kaufmann, and R.S. Raghavan, Phys. Rev. B13, 2835 (1976).

[4] Hyperfine Interaction Conference, Madison (1977).

[5] C. Domienikan, F. de Toledo, R.N. Saxena, A.W. Carbonari, and J.M. Filho, Progress Report 1997/1998 - IPEN/SP Pg 67.

[6] A.W. Carbonari and H. Haas, Hyp. Interactions 133, 71 (2001). 